\title{
Divergence Free QED Lagrangian in (2 + 1)-Dimensional Space-Time with Three Different Regularization Prescriptions
}

\author{
M. Forkan', M. Abul Mansur Chowdhury ${ }^{2}$ \\ ${ }^{1}$ Department of Mathematics, University of Chittagong, Chittagong, Bangladesh \\ ${ }^{2}$ JNI Research Centre for Mathematical and Physical Sciences, University of Chittagong, Chittagong, Bangladesh \\ Email: forkan.math@cu.ac.bd, mamansur@cu.ac.bd
}

How to cite this paper: Forkan, M. and Chowdhury, M.A.M. (2018) Divergence Free QED Lagrangian in $(2+1)$-Dimensional Space-Time with Three Different Regularization Prescriptions. Journal of Applied Mathematics and Physics, 6, 2067-2086. https://doi.org/10.4236/jamp.2018.610175

Received: June 11, 2018

Accepted: October 22, 2018

Published: October 25, 2018

Copyright ( $\odot 2018$ by authors and Scientific Research Publishing Inc. This work is licensed under the Creative Commons Attribution International License (CC BY 4.0).

http://creativecommons.org/licenses/by/4.0/ (c) (i) Open Access

\begin{abstract}
Quantum field theory can be understood through gauge theories. It is already established that the gauge theories can be studied either perturbatively or non-perturbatively. Perturbative means using Feynman diagrams and non-perturbative means using Path-integral method. Operator regularization $(\mathrm{OR})$ is one of the exceptional methods to study gauge theories because of its two-fold prescriptions. That means in OR two types of prescriptions have been introduced, which gives us the opportunity to check the result in self consistent way. In an earlier paper, we have evaluated basic QED loop diagrams in $(3+1)$ dimensions using the both methods of OR and Dimensional regularization (DR). Then all three results have been compared. It is seen that the finite part of the result is almost same. In this paper, we are interested to evaluate the same basic loop diagrams in $(2+1)$ space-time dimensions, because of two reasons: the main reason in $(2+1)$ space-time dimensions, these loops diagrams are finite, on other hand, there are divergences in $(3+1)$ space-time dimensions and the other reason is to see validity of using OR to evaluate Feynman loop diagrams in all dimensions. Here we have used both prescriptions of OR and DR to evaluate the basic loop diagrams and compared the results. Interestingly the results are almost same in all cases.
\end{abstract}

\section{Keywords}

Operator Regularization, Dimensional Regularization, Feynman Diagrams in QED, Path-Integral Method, Background Field Quantization and Generating Functional

\section{Introduction}

Gauge theories [1]-[7] describe the interactions of all known forces such as elec- 
tromagnetic, week and strong interations. To get a clear picture of different features from these theories, renormalization is a must. These can be done in two ways. That means one can study these theories either perturbatively or non-perturbatively. In the perturbative method, we mainly get some Feynman diagrams from the theory. When we evaluate loop diagrams in some cases, the loop integrals are divergent. So we need to regularize the integrals. That is why perturbative method needs regularization prescription. There are many regularization methods available in the literature. However, the most popular and appropriately applicable methods are Dimensional regularization (DR) [8] [9], Pauli-Villars regularization [10], Pre-regularization [11] [12] [13] [14] and some others. In non-perturbative method, we expand the generating function in terms of path integrals, then using different techniques we try to renormalize the theory to find the different features of the particles involved in the interactions. Operator regularization (OR) method is one of the best non-perturbative methods to study gauge theories. The remarkable feature of this method is that it gives us the opportunity to study the theory both perturbatively and non-perturbatively. That means although OR is mainly a path integral method but at one stage there is an option to consider the term as a factor for operator for loop diagrams. Then we can evaluate Feynman diagrams using these operators. Operator regularization method was prescribed by D.G.C. McKeon et al. [15] [16] [17] to study gauge theories non-perturbatively. However, they mentioned there that at one stage one can also use this prescription perturbatively. That means at that point one can also draw Feynman diagrams. A.Y. Sheiek [18] [19] has showed how one can use Feynman diagrammatic technique in OR method.

In an earlier paper [20] we have described OR method in both ways and evaluated one-loop Feynman diagrams in QED in $(3+1)$ space-time dimensions. Also we have used DR method in evaluating these diagrams and compared the results with the results obtained in OR in both ways. We have seen all the results are exactly same, except a finite constant term which will not affect the renormalization of the theory. In this paper, we have used the same method to evaluate the basic QED one-loop Feynman diagrams in $(2+1)$ space-time dimensions to see the basic difference between finite and infinite loop integrals. Because in $(3+$ 1) dimensions, the loop integrals are divergent, on the other hand, in $(2+1)$ dimensions, the loop integrals are finite.

\section{Operator Regularization Prescription}

Operator regularization is a convenient method of computing quantum corrections in quantum field theory in the context of background-field quantization and using path integral method [21] [22] [23], which were given by D.G.C. McKeon et al. In this method the Feynman diagrams of the usual perturbation series can be avoided. But at one stage there is an option to consider the factor used for operators and inverse operators for Feynman diagrams. That is from this prescription one can choose either path integral method or Feynman dia- 
grammatic approach.

We have clearly explained how this OR can be applied for evaluating Feynman diagrams in ref. [18]. For self consistence let us write a few main steps of this prescription which has to be used in evaluating the one-loop Feynman diagrams in $(2+1)$-dimensions.

In gauge theories we mainly deal with the generating functions. Then after some simplification we end up with some types operator and inverse operators. Then how one can take care of these operators has been explained in this OR.

If we have an operator $\Omega$ then according to OR we can write

$$
\operatorname{det} \Omega=\exp (\operatorname{tr} \ln \Omega)
$$

Let us regularize $\ln \Omega$ in the following way:

$$
\ln \Omega=-\lim _{\varepsilon \rightarrow 0} \frac{\mathrm{d}^{n}}{\mathrm{~d} \varepsilon^{n}}\left(\frac{\varepsilon^{n-1}}{n !} \Omega^{-\varepsilon}\right) \quad(n=1,2,3, \cdots)
$$

In facing no divergences we can always choose $n$ to be greater than or equal to the number of "loop momentum integrals" or in other words order in $\hbar$.

Hence,

$$
\operatorname{det} \Omega=\exp \left[\operatorname{tr}\left\{-\lim _{\varepsilon \rightarrow 0} \frac{\mathrm{d}^{n}}{\mathrm{~d} \varepsilon^{n}}\left(\frac{\varepsilon^{n-1}}{n !} \Omega^{-\varepsilon}\right)\right\}\right]
$$

and

$$
\begin{aligned}
\Omega^{-m} & =\frac{(-1)^{m-1}}{(m-1) !} \frac{\mathrm{d}^{m}}{\mathrm{~d} \Omega^{m}}(\ln \Omega) \\
& =-\lim _{\varepsilon \rightarrow 0} \frac{\mathrm{d}^{n}}{\mathrm{~d} \varepsilon^{n}}\left(\frac{\varepsilon^{n-1}}{n !} \frac{\Gamma(\varepsilon+m)}{\Gamma(m) \Gamma(\varepsilon)} \Omega^{-\varepsilon-m}\right)
\end{aligned}
$$

If we now rewrite $\Omega^{-\varepsilon}$ as

$$
\Omega^{-\varepsilon}=\frac{1}{\Gamma(\varepsilon)} \int_{0}^{\infty} \mathrm{d} t t^{\varepsilon-1} \exp (-\Omega t) .
$$

in Equation (2.9) we arrive at the result

$$
\operatorname{det} \Omega=\exp \left[-\xi^{\prime}(0)\right]
$$

where we have defined the $\xi$-function

$$
\xi(\varepsilon)=\frac{1}{\Gamma(\varepsilon)} \int_{0}^{\infty} \mathrm{d} t t^{\varepsilon-1} \operatorname{tr} \exp (-\Omega t)
$$

This is the usual $\xi$-function regularization of the determinant of an operator [24] [25].

Equations (2.2) and (2.3) are the main steps of the Operator regularization which has to be used in evaluating the Green's function of any problem. From this point we can divide the prescription in two fold way. That means if we want to follow the path integral method we have to use Schwinger expansion [26] for the operator like 


$$
\begin{aligned}
\operatorname{det} \Omega= & \exp \left\{-\lim _{\varepsilon \rightarrow 0} \frac{\mathrm{d}}{\mathrm{d} \varepsilon}\left[\frac { 1 } { \Gamma ( \varepsilon ) } \int _ { 0 } ^ { \infty } \mathrm { d } t t ^ { \varepsilon - 1 } \operatorname { t r } \left(\mathrm{e}^{-\Omega_{0} t}-t \mathrm{e}^{\Omega_{0} t} \Omega_{I} \frac{t^{2}}{2} \int_{0}^{1} \mathrm{~d} u \mathrm{e}^{-(1-u) \Omega_{0} t} \Omega_{I} \mathrm{e}^{-u \Omega_{0} t} \Omega_{I}\right.\right.\right. \\
& \left.\left.\left.-\frac{t^{3}}{3} \int_{0}^{1} \mathrm{~d} u u \int_{0}^{1} \mathrm{~d} v e^{-(1-u) \Omega_{0} t} \Omega_{I} \mathrm{e}^{-u(1-v) \Omega_{0} t} \Omega_{I} \mathrm{e}^{-u v \Omega_{0} t} \Omega_{I}+\cdots\right)\right]\right\}
\end{aligned}
$$

where, $\Omega=\Omega_{0}+\Omega_{I}$ with $\Omega_{0}$ is independent of the background field $f_{i}$ and $\Omega_{I}$ is at least linear in $f_{i}$.

Then following the steps described in ref. [15] we can find the result of the problems in consideration.

On the other hand if we want to use perturbatuve method then we have to take $n=1$ for one -loop, take $n=2$ for two-loops in Equations (2.2) and (2.3) and so on.

From Equation (2.3b) we can write the general prescription of Operator regularization for the Feynman diagrams following [18]:

$$
\Omega^{-m}=\lim _{\varepsilon \rightarrow 0} \frac{\mathrm{d}^{n}}{\mathrm{~d} \varepsilon^{n}}\left[\left(1+\alpha_{1} \varepsilon+\alpha_{2} \varepsilon^{2}+\cdots+\alpha_{n} \varepsilon^{n}\right) \frac{\varepsilon^{n}}{n !} \Omega^{-\varepsilon-m}\right]
$$

where the $\alpha_{n}$ s are arbitrary. For one-loop diagrams it is enough to use $n=1$. When $m=2$ and $n=1$, then Equation (2.7) taken the form

$$
\Omega^{-2}=\lim _{\varepsilon \rightarrow 0} \frac{\mathrm{d}}{\mathrm{d} \varepsilon}\left[\varepsilon(1+\alpha \varepsilon) \Omega^{-\varepsilon-2}\right]
$$

In one loop calculations we can use (2.8) for operators. In the following sections we will use this prescription for evaluating the three basic one loop diagrams.

\subsection{One Loop Fermions Correction in $(2+1)$ Dim. Using Dimensional and Operator Regularizations [18] [27] [28] [29]}

\section{1) Dimensional Regularization Method:}

Starting with the Feynman diagram for the one loop correction to the fermions line shown in Figure 1 which is represented by $\left(\sum(p)\right)$ :

Using the standard Feynman rules one can write $\left(\sum(p)\right)$ as,

$$
\left(\sum(p)\right)=-i e^{2} \int \frac{\mathrm{d}^{3} l}{(2 \pi)^{3}} \gamma_{\mu} \frac{(p-l-m)}{\left[(p-l)^{2}-m^{2}\right] l^{2}} \gamma_{\mu}
$$

Using the Feynman identity for combining the denominators, we can write

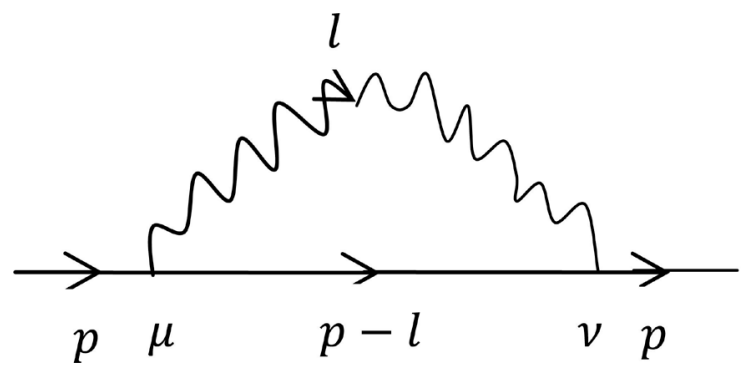

Figure 1. One loop Feynman diagram for external fermions lines. 


$$
\left(\sum(p)\right)=-i e^{2} \int_{0}^{1} \mathrm{~d} x \int \frac{\mathrm{d}^{3} l}{(2 \pi)^{3}} \frac{\gamma_{\mu}(p-l-m) \gamma_{\mu}}{\left[(p-l)^{2} x-m^{2} x+l^{2}(1-x)\right]^{2}}
$$

Shifting the variable of integration as $l^{\prime}=l-p x$ and simplifying we get

$$
\left(\sum(p)\right)=-i e^{2} \int_{0}^{1} \mathrm{~d} x \int \frac{\mathrm{d}^{3} l^{\prime}}{(2 \pi)^{3}} \frac{\gamma_{\mu}\left[p(1-x)-m-l^{\prime}\right] \gamma_{\mu}}{\left[l^{\prime 2}-m^{2} x+p^{2} x(1-x)\right]^{2}}
$$

The term linear in $l^{\prime}$ integrates to zero because of symmetric integration, so

$$
\left(\sum(p)\right)=-i e^{2} \int_{0}^{1} \mathrm{~d} x \int \frac{\mathrm{d}^{3} l}{(2 \pi)^{3}} \frac{\gamma_{\mu}[p(1-x)-m] \gamma_{\mu}}{\left[l^{2}-m^{2} x+p^{2} x(1-x)\right]^{2}}
$$

Which is taken as the common starting point for both Dimensional and Operator regularization.

Using Feynman identity and then $\gamma$-algebra, the above result becomes,

$$
\begin{aligned}
\left(\sum(p)\right) & =\frac{e^{2} \mu \sqrt{\pi}}{(4 \pi)^{2}}(2 \sqrt{\pi} \mu) \int_{0}^{1} \mathrm{~d} x \frac{[p(1-x)+3 m]}{\left[m^{2}-p^{2} x(1-x)\right]^{1 / 2}} \\
& =\frac{e^{2} \mu^{2}}{(8 \pi)} \int_{0}^{1} \mathrm{~d} x \frac{[p(1-x)+3 m]}{\left[m^{2}-p^{2} x(1-x)\right]^{1 / 2}}
\end{aligned}
$$

Thus according to dimensional regularization, we see that there is no divergent part in $(2+1)$-dimensional space-time, because the integrals are finite in 3-dimensions.

\section{2) Operator Regularization Method:}

The same one-loop correction to fermion can be evaluated using OR, following the rule cited in Equations (2.5) and (2.6) in ref. [8]. The amplitude of the self-energy diagram as

$$
\begin{aligned}
& \left(\sum(p)\right) \\
& =-i e^{2} \int_{0}^{1} \mathrm{~d} x \lim _{\varepsilon \rightarrow 0} \frac{\mathrm{d}}{\mathrm{d} \varepsilon} \int \frac{\mathrm{d}^{3} l}{(2 \pi)^{3}} \frac{\varepsilon(1+\alpha \varepsilon) \gamma_{\mu}[p(1-x)-m] \gamma_{\mu}}{\left[l^{2}-m^{2}+p^{2} x(1-x)\right]^{\varepsilon+2}}
\end{aligned}
$$

Using the standard integral

$$
\int \frac{\mathrm{d}^{2 w} l}{(2 \pi)^{2 w}} \frac{1}{\left(l^{2}+M^{2}\right)^{A}}=\frac{1}{(4 \pi)^{w} \Gamma(A)} \frac{\Gamma(A-w)}{\left(M^{2}\right)^{A-w}}
$$

we get,

$$
\begin{aligned}
&\left(\sum(p)\right)= \frac{-i e^{2}}{(4 \pi)^{3 / 2}} \int_{0}^{1} \mathrm{~d} x \gamma_{\mu}[p(1-x)-m] \gamma_{\mu} \\
& \cdot \lim _{\varepsilon \rightarrow 0} \frac{\mathrm{d}}{\mathrm{d} \varepsilon}\left[\frac{\varepsilon(1+\alpha \varepsilon)}{\Gamma(\varepsilon+2)} \cdot \frac{\Gamma\left(\varepsilon+\frac{1}{2}\right)}{\left(-m^{2} x+p^{2} x(1-x)\right)^{\varepsilon+\frac{1}{2}}}\right]
\end{aligned}
$$




$$
\text { Here, } \begin{aligned}
\lim _{\varepsilon \rightarrow 0} \frac{\mathrm{d}}{\mathrm{d} \varepsilon}\left[\frac{\varepsilon(1+\alpha \varepsilon)}{\Gamma(\varepsilon+2)} \cdot \frac{\Gamma\left(\varepsilon+\frac{1}{2}\right)}{\left(-m^{2} x+p^{2} x(1-x)\right)^{\varepsilon+\frac{1}{2}}}\right] \\
=\lim _{\varepsilon \rightarrow 0} \frac{\mathrm{d}}{\mathrm{d} \varepsilon}\left[\frac{\varepsilon(1+\alpha \varepsilon)\left(\varepsilon-\frac{1}{2}\right) ! u^{-\left(\varepsilon+\frac{1}{2}\right)}}{(\varepsilon+1) !}\right]
\end{aligned}
$$

where $u=-m^{2} x+p^{2} x(1-x)$

$$
\begin{aligned}
& =\lim _{\varepsilon \rightarrow 0}\left[\frac{u^{-\left(\varepsilon+\frac{1}{2}\right)}(\varepsilon \alpha)\left(-\frac{1}{2}+\varepsilon\right)}{(1+\varepsilon) !}+\frac{u^{-\left(\varepsilon+\frac{1}{2}\right)}(1+\alpha \varepsilon)\left(\varepsilon-\frac{1}{2}\right) !}{(1+\varepsilon) !}\right. \\
& -\frac{u^{-\left(\varepsilon+\frac{1}{2}\right)} \varepsilon(1+\alpha \varepsilon)\left(\varepsilon-\frac{1}{2}\right) ! \ln u}{(1+\varepsilon) !}+\frac{u^{-\left(\varepsilon+\frac{1}{2}\right)} \varepsilon(1+\varepsilon \alpha) \Gamma\left(\varepsilon+\frac{1}{2}\right) F\left(\varepsilon+\frac{1}{2}\right)}{(1+\varepsilon) !} \\
& \left.-\frac{u^{-\left(\varepsilon+\frac{1}{2}\right)} \varepsilon(1+\varepsilon \alpha)\left(\varepsilon-\frac{1}{2}\right) ! \Gamma(\varepsilon+2) F(\varepsilon+2)}{(1+\varepsilon) !}\right]=\frac{\sqrt{\pi}}{u^{1 / 2}}
\end{aligned}
$$

Therefore,

$$
\lim _{\varepsilon \rightarrow 0} \frac{\mathrm{d}}{\mathrm{d} \varepsilon}\left[\frac{\varepsilon(1+\alpha \varepsilon)}{\Gamma(\varepsilon+2)} \cdot \frac{\Gamma\left(\varepsilon+\frac{1}{2}\right)}{\left(-m^{2} x+p^{2} x(1-x)\right)^{\varepsilon+\frac{1}{2}}}\right]=\frac{\sqrt{\pi}}{\left[p^{2} x(1-x)-m^{2} x\right]^{1 / 2}}
$$

Thus Equation (2.1.4) becomes,

$$
\begin{aligned}
\left(\sum(p)\right) & =\frac{-i e^{2}}{(4 \pi)^{3 / 2}} \int_{0}^{1} \mathrm{~d} x[p(1-x)+3 m] \frac{\sqrt{\pi}}{\left[p^{2} x(1-x)-m^{2} x\right]^{1 / 2}} \\
& =\frac{-e^{2}}{(8 \pi)} \int_{0}^{1} \mathrm{~d} x \frac{[p(1-x)+3 m]}{\left[m^{2}-p^{2} x(1-x)\right]^{1 / 2}}
\end{aligned}
$$

This is the same form as like as obtained by dimensional regularization approach.

\subsection{One Loop Photon Correction in $(2+1)$ Dim. Using Dimensional and Operator Regularizations}

Let us consider the Feynman diagram for the one loop correction to the photon line shown in Figure 2 which is represented by $\Pi_{\mu v}(p)$ :

The QED one loop correction to the photon line in $(2+1)$-dimensions is

$$
\Pi_{\mu v}(p)=e^{2} \int \frac{\mathrm{d}^{3} l}{(2 \pi)^{3}} \operatorname{Tr}\left[\frac{\gamma_{\mu}(l+p-m) \gamma_{\nu}(l-m)}{\left[(l+p)^{2}-m^{2}\right]\left(l^{2}-m^{2}\right)}\right]
$$




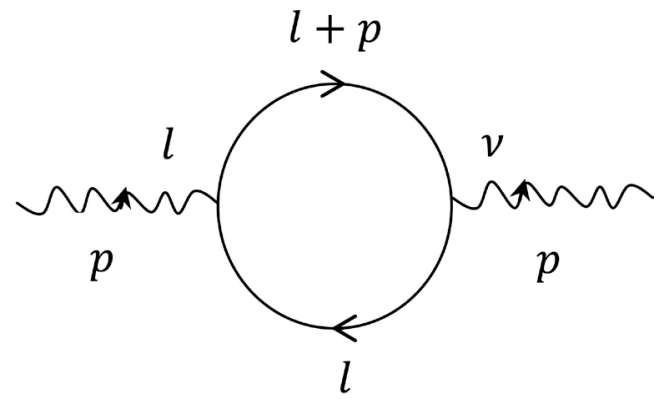

Figure 2. One loop Feynman diagram for external boson lines.

Combining the denominator using the Feynman identity and simplifying, we get

$$
\Pi_{\mu \nu}(p)=e^{2} \int_{0}^{1} \mathrm{~d} x \int \frac{\mathrm{d}^{3} l}{(2 \pi)^{3}} \frac{\operatorname{Tr}\left[\gamma_{\mu}(l+p-m) \gamma_{v}(l-m)\right]}{\left[(l+p)^{2} x-m^{2} x+\left(l^{2}-m^{2}\right)(1-x)\right]^{2}}
$$

Now putting $l^{\prime}=l+p x$ in Equation (2.2.1), then we get,

$$
\Pi_{\mu \nu}(p)=e^{2} \int_{0}^{1} \mathrm{~d} x \int \frac{\mathrm{d}^{3} l^{\prime}}{(2 \pi)^{3}} \frac{\operatorname{Tr}\left[\gamma_{\mu}\left(l^{\prime}-p x+p-m\right) \gamma_{v}\left(l^{\prime}-p x-m\right)\right]}{\left[\left(l^{\prime}+p(1-x)\right)^{2} x-m^{2} x+\left(\left(l^{\prime}-p x\right)^{2}-m^{2}\right)(1-x)\right]^{2}}
$$

After simplification Equation (2.2.1) with $l^{\prime} \rightarrow l$ becomes,

$$
\begin{aligned}
\Pi_{\mu v}(p)= & 4 e^{2} \int_{0}^{1} \mathrm{~d} x \int \frac{\mathrm{d}^{3} l}{(2 \pi)^{3}}\left[\frac{2 l_{\mu} l_{v}}{\left\{l^{2}-m^{2}+p^{2} x(1-x)\right\}^{2}}\right. \\
& \left.-\frac{2 x(1-x)\left(p_{\mu} p_{v}-p^{2} \delta_{\mu v}\right)}{\left\{l^{2}-m^{2}+p^{2} x(1-x)\right\}^{2}}-\frac{\delta_{\mu v}}{\left\{l^{2}-m^{2}+p^{2} x(1-x)\right\}}\right]
\end{aligned}
$$

If we apply the following integrals in the first and third terms in the integrand of Equation (2.2.2),

$$
\begin{aligned}
\text { I) } & \int \mathrm{d}^{d} l \frac{l_{\mu} l_{v}}{\left(l^{2}+2 l q-m^{2}\right)^{\alpha}} \\
& =\frac{i \pi^{d / 2}}{\alpha} \frac{1}{\left(-q^{2}-m^{2}\right)^{\alpha-d / 2}}\left[q_{\mu} q_{v} \Gamma \alpha-\frac{d}{2}+\frac{1}{2} g_{\mu v}\left(-q^{2}-m^{2}\right) \Gamma\left(\alpha-1-\frac{d}{2}\right)\right]
\end{aligned}
$$

II) $\int \mathrm{d}^{d} l \frac{1}{\left(l^{2}+2 l q-m^{2}\right)^{\alpha}}=(-1)^{\alpha} i \pi^{d / 2} \frac{\Gamma(\alpha-d / 2)}{\Gamma(\alpha)\left(-q^{2}-m^{2}\right)^{\alpha-d / 2}}$

We arrive at,

$$
\Pi_{\mu \nu}(p)=8 e^{2}\left(p_{\mu} p_{v}-p^{2} \delta_{\mu \nu}\right) \int_{0}^{1} \mathrm{~d} x \int \frac{\mathrm{d}^{3} l}{(2 \pi)^{3}}\left[\frac{x(1-x)}{\left\{l^{2}-m^{2}+p^{2} x(1-x)\right\}^{2}}\right]
$$

Which is again taken as the common starting point for both Dimensional and Operator regularization for one loop correction to the photon lines.

Using Feynman identity-II, the above result becomes, 


$$
\begin{aligned}
\Pi_{\mu v}(p) & =\frac{-8 i e^{2}\left(p_{\mu} p_{v}-\delta_{\mu v} p^{2}\right)}{(2 \pi)^{3 / 2}} \int_{0}^{1} \mathrm{~d} x x(1-x)\left[\frac{p^{2} x(1-x)-m^{2}}{2 \pi \mu^{2}}\right]^{-\varepsilon / 2} \\
& =\frac{-8 i e^{2} \mu\left(p_{\mu} p_{v}-\delta_{\mu v} p^{2}\right)}{(2 \pi)} \int_{0}^{1} \mathrm{~d} x \frac{x(1-x)}{\left[p^{2} x(1-x)-m^{2}\right]^{1 / 2}}
\end{aligned}
$$

Thus according to dimensional regularization, we see that there is no divergent part in $(2+1)$-dimensional space-time, because the integrals are finite in 3-dimensions.

Now proceeding with operator regularization and again following the same route, we get,

$\Pi_{\mu \nu}(p)=-8 e^{2}\left(p_{\mu} p_{v}-p^{2} \delta_{\mu \nu}\right) \int_{0}^{1} \mathrm{~d} x \lim _{\varepsilon \rightarrow 0} \frac{\mathrm{d}}{\mathrm{d} \varepsilon} \int \frac{\mathrm{d}^{3} l}{(2 \pi)^{3}} \frac{\varepsilon(1+\alpha \varepsilon) x(1-x)}{\left[l^{2}-m^{2}+p^{2} x(1-x)\right]^{\varepsilon+2}}$

Performing the momentum integral (2.1.3), so that relative to the Equation (2.2.5) we get, $A=\varepsilon+2, w=\frac{3}{2}, M^{2}=-m^{2}+p^{2} x(1-x)$, then Equation (2.2.5) becomes,

$$
\begin{array}{r}
\Pi_{\mu v}(p)=-8 e^{2} \frac{1}{(4 \pi)^{3 / 2}}\left(p_{\mu} p_{v}-p^{2} \delta_{\mu \nu}\right) \int_{0}^{1} \mathrm{~d} x x(1-x) \\
\cdot \lim _{\varepsilon \rightarrow 0} \frac{\mathrm{d}}{\mathrm{d} \varepsilon}\left[\frac{\varepsilon(1+\alpha \varepsilon)}{\Gamma(\varepsilon+2)} \cdot \frac{\Gamma\left(\varepsilon+\frac{1}{2}\right)}{\left(-m^{2}+p^{2} x(1-x)\right)^{\varepsilon+\frac{1}{2}}}\right]
\end{array}
$$

Now using the Equation (2.1.4) then above equation reduces to,

$$
\begin{aligned}
\Pi_{\mu \nu}(p) & =-8 e^{2} \frac{1}{(4 \pi)^{3 / 2}}\left(p_{\mu} p_{v}-p^{2} \delta_{\mu \nu}\right) x(1-x) \frac{\sqrt{\pi}}{\left[p^{2} x(1-x)-m^{2} x\right]^{1 / 2}} \\
& =\frac{-4 e^{2}\left(p_{\mu} p_{v}-\delta_{\mu \nu} p^{2}\right)}{(2 \pi)} \int_{0}^{1} \mathrm{~d} x \frac{x(1-x)}{\left[p^{2} x(1-x)-m^{2}\right]^{1 / 2}}
\end{aligned}
$$

This is the same form as we obtained by dimensional regularization approach.

\subsection{One Loop Vertex Correction in $(2+1)$ Dim. Using Dimensional and Operator Regularizations}

Let us now consider the Feynman diagram for the one loop correction to the vertex shown in Figure 3 which is represented by $\Gamma_{\rho}(p, q)$.

The QED one loop correction to the vertex in $(2+1)$-dimensions is

$$
\begin{aligned}
\Gamma_{\rho}(p, q) & =\int \frac{\mathrm{d}^{3} l}{(2 \pi)^{3}}\left[\left(-i e \gamma_{\lambda}\right) \frac{i}{(p+l+m)}\left(-i e \gamma_{\rho}\right) \frac{i}{q+l+m}\left(-i e \gamma_{\sigma}\right) \frac{\delta_{\tau \sigma}}{l^{2}}\right] \\
& =-i e^{3} \int \frac{\mathrm{d}^{3} l}{(2 \pi)^{3}} \frac{\left[\gamma_{\lambda}(p+l-m) \gamma_{\rho}(q+l-m) \gamma_{\lambda}\right]}{l^{2}\left[(p+l)^{2}-m^{2}\right]\left[(q+l)^{2}-m^{2}\right]}
\end{aligned}
$$

Applying the 3-parameter Feynman formula for combining the denominator 


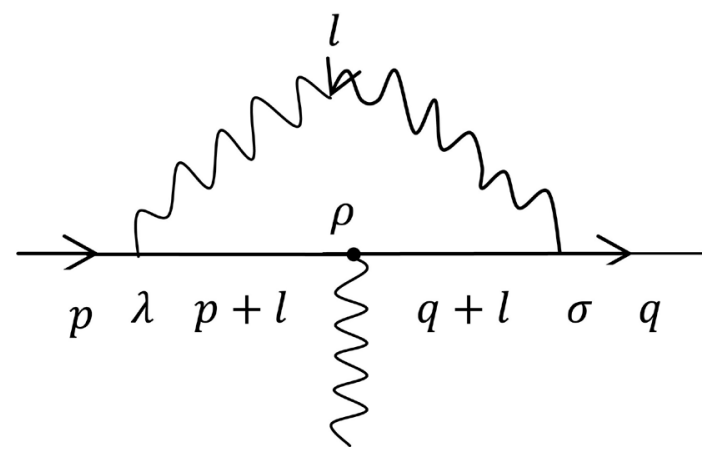

Figure 3. One loop Feynman diagram for vertex function.

and shifting the variable of integration $l \rightarrow l+p x+q y$ and simplify the denominator and numerator, we obtain,

$$
\begin{aligned}
\Gamma_{\rho}(p, q)= & -2 i e^{3} \int_{0}^{1} \mathrm{~d} x \int_{0}^{1-x} \mathrm{~d} y \int \frac{\mathrm{d}^{3} l}{(2 \pi)^{3}} \\
& \frac{\left[\gamma_{\lambda}(l+p(1+x)+q y-m) \gamma_{\rho}(l+q(1+y)+p x-m) \gamma_{\lambda}\right]}{\left[l^{2}-m^{2}(x+y)+p^{2} x(1-x)+q^{2} y(1-y)-2 p \cdot q x y\right]^{3}}
\end{aligned}
$$

This integral contains convergent and divergent pieces. The part of the numerator quadratic in $l$ is divergent, the rest convergent, so separating the divergent piece $\Gamma_{\rho}^{(1)}(p, q)$ and convergent piece $\Gamma_{\rho}^{(2)}(p, q)$, i.e.

$$
\Gamma_{\rho}(p, q)=\Gamma_{\rho}^{(1)}(p, q)+\Gamma_{\rho}^{(2)}(p, q)
$$

Thus the divergent piece is,

$$
\Gamma_{\rho}^{(1)}(p, q)=-2 i e^{3} \int_{0}^{1} \mathrm{~d} x \int_{0}^{1-x} \mathrm{~d} y \int \frac{\mathrm{d}^{3} l}{(2 \pi)^{3}} \frac{\gamma_{\sigma} l \gamma_{\rho} l \gamma_{\sigma}}{\left(l^{2}-M^{2}\right)^{3}}
$$

where, $M^{2} \equiv m^{2}(x+y)-p^{2} x(1-x)-q^{2} y(1-y)+2 p \cdot q x y$.

Which is taken as the common starting point for both Dimensional and Operator regularizations for one-loop correction to the vertex.

Using Feynman identity and then $\gamma$-algebra, the above result becomes,

$$
\begin{aligned}
\Gamma_{\rho}^{(1)}(p, q) & =\frac{2 e^{3} \mu \Gamma\left(\frac{1}{2}\right)}{(4 \pi)^{2}} \int_{0}^{1} \mathrm{~d} x \int_{0}^{1-x} \mathrm{~d} y \frac{\gamma_{\rho}}{\left(M^{2}\right)^{1 / 2}} \\
& =\frac{2 e^{3} \mu \sqrt{\pi} \gamma_{\rho}}{(4 \pi)^{2}} \int_{0}^{1} \mathrm{~d} x \int_{0}^{1-x} \mathrm{~d} y \frac{1}{\left(M^{2}\right)^{1 / 2}} \\
& =\frac{e^{3} \mu \gamma_{\rho}}{(4 \pi)} \int_{0}^{1} \mathrm{~d} x \int_{0}^{1-x} \mathrm{~d} y \frac{1}{\left(M^{2}\right)^{1 / 2}}
\end{aligned}
$$

where, $M^{2} \equiv m^{2}(x+y)-p^{2} x(1-x)-q^{2} y(1-y)+2 p \cdot q x y$.

Now proceeding with operator regularization through the rule (2.13) and (2.14), we get, 


$$
\Gamma_{\rho}^{(1)}(p, q)=-2 i e^{3} \int_{0}^{1} \mathrm{~d} x \int_{0}^{1-x} \mathrm{~d} y \lim _{\varepsilon \rightarrow 0} \frac{\mathrm{d}}{\mathrm{d} \varepsilon} \int \frac{\mathrm{d}^{3} l}{(2 \pi)^{3}} \varepsilon(1+\alpha \varepsilon) \frac{\gamma_{\sigma} l \gamma_{\rho} l \gamma_{\sigma}}{\left(l^{2}-M^{2}\right)^{\varepsilon+3}}
$$

Now performing the momentum integral -I from above, then we get,

$$
\Gamma_{\rho}^{(1)}(p, q)=-i e \frac{e^{2}}{(4 \pi)^{3 / 2}} \int_{0}^{1} \mathrm{~d} x \int_{0}^{1-x} \mathrm{~d} y \lim _{\varepsilon \rightarrow 0} \frac{\mathrm{d}}{\mathrm{d} \varepsilon}\left[\frac{\varepsilon(1+\alpha \varepsilon)}{\Gamma(\varepsilon+3)} \cdot \frac{\Gamma\left(\varepsilon+\frac{1}{2}\right)}{\left(M^{2}\right)^{\varepsilon+\frac{1}{2}}}\right] \gamma_{\sigma} \gamma_{\lambda} \gamma_{\rho} \gamma_{\tau} \gamma_{\sigma}
$$

Now using the Equation (2.1.4) and $\gamma$-algebra, then above equation reduces to,

$\Gamma_{\rho}^{(1)}(p, q)=-i e \frac{e^{2}}{(4 \pi)^{3 / 2}} \int_{0}^{1} \mathrm{~d} x \int_{0}^{1-x} \mathrm{~d} y \frac{\sqrt{\pi}}{2\left[M^{2}\right]^{1 / 2}} \gamma_{\rho}=-i e \frac{e^{2} \gamma_{\rho}}{(16 \pi)} \int_{0}^{1} \mathrm{~d} x \int_{0}^{1-x} \mathrm{~d} y \frac{1}{\left[M^{2}\right]^{1 / 2}}$

where, $M^{2} \equiv m^{2}(x+y)-p^{2} x(1-x)-q^{2} y(1-y)+2 p \cdot q x y$.

This is the same form as like as obtained by dimensional regularization approach.

\section{Path Integral Form of Operator Regularization for One Loop Generating Functional in QED}

The path integral form of OR for one-loop case is described in ref. [15]. That is if we consider the QED Lagrangian as,

$$
L=-\frac{1}{4}\left(\partial_{\mu} \Omega_{\mu}-\partial_{\nu} \Omega_{v}\right)^{2}+\bar{\Psi}\left(-i \gamma^{\mu} \partial_{\mu}-e \gamma^{\mu} \Omega_{\mu}-m\right) \Psi-\frac{1}{2 \alpha}\left(\partial_{\mu} \Omega^{\mu}\right)^{2}
$$

and let us expand this Lagrangian taking background field quantization of the fields in the following form, gauge field $\Omega_{\mu}$ and fermionic field $\psi$ are respectively,

$$
\begin{aligned}
& \Omega_{\mu}=V_{\mu}+Q_{\mu}, \text { for gauge field } \Omega_{\mu} \\
& \psi=\eta+q, \text { for fermionic field } \psi,
\end{aligned}
$$

where $V_{\mu}$ and $\eta$ are the classical fields and $Q_{\mu}$ and $q$ are the quantum fields.

Therefore Equation (3.1) becomes,

$$
\begin{aligned}
L=- & \frac{1}{4}\left(\partial_{\mu} V_{\mu}+\partial_{\mu} Q_{\mu}-\partial_{v} V_{v}-\partial_{\nu} Q_{v}\right)^{2} \\
+ & (\bar{\eta}+\bar{q})\left(-i \gamma^{\mu} \partial_{\mu}-e \gamma^{\mu}\left(V_{\mu}+Q_{\mu}\right)-m\right)(\eta+q) \\
- & \frac{1}{2 \alpha}\left(\partial_{\mu} V_{\mu}+\partial_{\mu} Q_{\mu}\right)^{2} \\
= & \frac{1}{4}\left[\left(\partial_{\mu} Q_{\mu}-\partial_{\nu} Q_{v}\right)+\left(\partial_{\mu} V_{\mu}-\partial_{\nu} V_{v}\right)\right]^{2}+\bar{\eta}\left(-i \gamma^{\mu} \partial_{\mu}-m\right) \eta \\
& +\bar{\eta}\left(-i \gamma^{\mu} \partial_{\mu}-m\right) q+\bar{q}\left(-i \gamma^{\mu} \partial_{\mu}-m\right) \eta \\
& +e\left(\bar{\eta} \gamma^{\mu} V_{\mu} \eta+\bar{\eta} \gamma^{\mu} V_{\mu} q+\bar{\eta} \gamma^{\mu} Q_{\mu} \eta+\bar{\eta} \gamma^{\mu} Q_{\mu} q+\bar{q} \gamma^{\mu} V_{\mu} \eta\right. \\
& \left.+\bar{q} \gamma^{\mu} V_{\mu} q+\bar{q} \gamma^{\mu} Q_{\mu} \eta+\bar{q} \gamma^{\mu} Q_{\mu} q\right)-\frac{1}{2 \alpha}\left(\partial_{\mu} V_{\mu}+\partial_{\mu} Q_{\mu}\right)^{2}
\end{aligned}
$$


Following ref. [7] the one-loop generating functional for Green's functions $Z_{1}$ is

$Z_{1}=\frac{\operatorname{det} D}{\operatorname{det}^{1 / 2}\left[p^{2} \delta_{\mu \nu}-\left(1-\frac{1}{\alpha}\right) p_{\mu} p_{\nu}-e^{2} \bar{\eta} \gamma_{\mu} D^{-1} \gamma_{\nu} \eta-e^{2} \bar{\eta} \gamma_{\nu} D^{-1} \gamma_{\mu} \eta\right]}=\frac{\operatorname{det} A}{\operatorname{det} B}$ (say)

where, $\mathbb{D}=\gamma^{\mu}\left(-i \partial_{\mu}-e V_{\mu}\right)=p-e V$

Here we see that $Z_{1}$ is the ratio of determinant of operators. Each of the determinants occurring in Equation (3.3) requires regularization and a corresponding $\xi$-function. The numerator and denominator separately contribute to Green's functions with only external boson lines and with both external fermions lines and vertex function in massless QED respectively.

\subsection{One-Loop Generating Functional and Loop Corrections for External Boson Lines}

To find the loop corrections or to write the generating functional for external boson lines one has to make a close look at the numerator of Equation (3.3) and on the other hand for external fermion lines one has to take care of the denominator of Equation (3.3). So for bosonic case we have to regulate the $\operatorname{det} A$ through the use of $\xi$-function in Equation (2.5a) yielding

$$
\operatorname{det} A=Z_{1}^{A}=\exp \left[-\lim _{\varepsilon \rightarrow 0} \xi^{\prime^{A}}(\varepsilon)\right]
$$

where,

$$
\xi(\varepsilon)=\frac{1}{\Gamma(\varepsilon)} \int_{0}^{\infty} \mathrm{d} t t^{\varepsilon-1} \operatorname{tr} \exp (-\Omega t) \text { with } \Omega=p-e V .
$$

As we mentioned in Section-2, after regularization we have to consider Schwinger expansion, to this view let us now identify the operator $\Omega_{0}$ and $\Omega_{I}$ with $p$ and $-e V$, respectively, then by Equation (2.4), Equation (3.1.1) can be written as,

$$
\begin{aligned}
Z_{1}^{A}= & \exp \left[-\lim _{\varepsilon \rightarrow 0} \frac{\mathrm{d}}{\mathrm{d} \varepsilon}\left\{\frac { 1 } { \Gamma ( \varepsilon ) } \int _ { 0 } ^ { \infty } \mathrm { d } t t ^ { \varepsilon - 1 } t r \left(e^{-p t}-t e^{-p t}+(-e V) \frac{t^{2}}{2}\right.\right.\right. \\
& \cdot \int_{0}^{1} \mathrm{~d} u e^{-(1-u) p t}(-e V) e^{-u p t}(-e V) \\
& \left.\left.\left.-\frac{t^{3}}{3} \int_{0}^{1} \mathrm{~d} u u \int_{0}^{1} \mathrm{~d} v e^{-(1-u) p t}(-e V) e^{-u(1-v) p t}(-e V) e^{-u v p t}(-e V)+\cdots\right)\right\}\right]
\end{aligned}
$$

To one-loop order this series plays the same role as Feynman rules in the usual perturbation theory. Here we want to evaluate the one-loop correction to the two-point function for external photon in QED; we restrict our attention to the term bilinear in $V_{\mu}$ on the right-hand side of Equation (3.1.3). This leaves us with

$$
Z_{1 V V}^{A}=\exp \left[-\lim _{\varepsilon \rightarrow 0} \frac{\mathrm{d}}{\mathrm{d} \varepsilon}\left\{\frac{e^{2}}{\Gamma(\varepsilon)} \int_{0}^{\infty} \mathrm{d} t \frac{t^{\varepsilon+1}}{2} \operatorname{tr}\left(\int_{0}^{1} \mathrm{~d} u e^{-(1-u) p t} V e^{-u p t} V\right)\right\}\right]
$$


Now let us complete the functional trace

$$
T=\operatorname{tr}\left(\int_{0}^{1} \mathrm{~d} u e^{-(1-u) p t} V e^{-u p t} V\right)
$$

Schwinger has pointed out that such traces are most easily evaluated in momentum space. We introduce a complete orthonormal set of states $|p\rangle$ that are eigenstates of the operator $p_{\mu}$, where, in $\mathrm{n}$ dimensions,

$$
\langle x \mid p\rangle=\frac{e^{i p \cdot x}}{(2 \pi)^{n / 2}}
$$

and

$$
\langle p|f| q\rangle=\frac{f(p-q)}{(2 \pi)^{n / 2}}
$$

On the right-hand side of Equation (3.1.6b), $f(p-q)$ is the Fourier transform of $f(x)$ :

$$
f(p-q)=\int \frac{\mathrm{d}^{n} x}{(2 \pi)^{n / 2}} f(x) e^{-i x \cdot(p-q)}
$$

Equation (3.1.5) takes the form,

$$
T=\int \mathrm{d}^{3} p \mathrm{~d}^{3} q \mathrm{~d}^{3} r \mathrm{~d}^{3} \$\left\langle p\left|e^{-(1-u) p t}\right| q\right\rangle\langle q|V| r\rangle\left\langle r\left|e^{-u p t}\right| \$\right\rangle\langle \$|V| p\rangle
$$

Upon inserting the complete set $1=\int \mathrm{d}^{3} p|p\rangle\langle p|$ at the appropriate places, and using (3.1.6), we rewrite Equation (3.1.8) as,

$$
\begin{aligned}
T= & \int \mathrm{d}^{3} p \mathrm{~d}^{3} q \mathrm{~d}^{3} r \mathrm{~d}^{3} \$ \frac{V(p-q)}{(2 \pi)^{3 / 2}} e^{-(1-u) p t} \cdot \frac{e^{i t \cdot q}}{(2 \pi)^{3 / 2}} \delta(\not r-q) \\
& \cdot \frac{V(\not-\$)}{(2 \pi)^{3 / 2}} e^{-u r t} \cdot \frac{e^{i \xi \cdot p}}{(2 \pi)^{3 / 2}} \delta(\$-p) \\
= & \int \frac{\mathrm{d}^{3} p \mathrm{~d}^{3} q}{(2 \pi)^{3}} e^{-(1-u) p t-u q t} V(p-q) V(q-p)
\end{aligned}
$$

After shifting the variable of integration $p \rightarrow p p+q$, Equation (3.1.9) becomes,

$$
T=\int \frac{\mathrm{d}^{3} p \mathrm{~d}^{3} q}{(2 \pi)^{3}} e^{-[q+(1-u) p] t} V(p) V(-p)
$$

Upon substituting Equation (3.1.10) into Equation (3.1.4), we find that

$$
Z_{1 V V}^{A}=\exp \left[-\lim _{\varepsilon \rightarrow 0} \xi_{V V}^{\prime A}(\varepsilon)\right]
$$

where,

$$
\xi_{V V}^{A}(\varepsilon)=\frac{e^{2}}{2 \Gamma(\varepsilon)} \int_{0}^{\infty} \mathrm{d} t t^{\varepsilon+1} \int_{0}^{1} \mathrm{~d} u \int \mathrm{d}^{3} p V(p) V(-p) \int \frac{\mathrm{d}^{3} q}{(2 \pi)^{3}} e^{-[q+(1-u) p] t}
$$

We use Equation (2.4) to integrate over $t$, then (3.1.11b) becomes, 


$$
\xi_{V V}^{A}(\varepsilon)=\frac{e^{2} \Gamma(\varepsilon+2)}{2 \Gamma(\varepsilon)} \int \mathrm{d}^{3} p V(p) V(-p) \int_{0}^{1} \mathrm{~d} u \int \frac{\mathrm{d}^{3} q}{(2 \pi)^{3}} \frac{[q-(1-u) p]^{\varepsilon+2}}{\left[q^{2}-(1-u)^{2} p^{2}\right]^{\varepsilon+2}}
$$

Now the last integral $I_{1}$ (say) of Equation (3.1.12) can be calculated as follows:

$$
\begin{aligned}
I_{1} & =\int \frac{\mathrm{d}^{3} q}{(2 \pi)^{3}} \frac{[q-(1-u) p]^{\varepsilon+2}}{\left[q^{2}-(1-u)^{2} p^{2}\right]^{\varepsilon+2}} \\
& =\int \frac{\mathrm{d}^{3} q}{(2 \pi)^{3}} \frac{\left[q^{\epsilon+2}-(\varepsilon+2)(q)^{\varepsilon+1}(1-u) p+\cdots+(1-u)^{\varepsilon+2}(p)^{\varepsilon+2}\right]}{\left[q^{2}-(1-u)^{2} p^{2}\right]^{\varepsilon+2}}
\end{aligned}
$$

Differentiating Equation (3.1.12) with respect to $\varepsilon$ and taking $\varepsilon \rightarrow 0$, we see that the product terms in $\varepsilon$ will vanish. Hence in the numerator of $I_{1}$ only the first and last term will contribute.

$$
\therefore I_{1}=\int \frac{\mathrm{d}^{3} q}{(2 \pi)^{3}}\left[\frac{\left(q^{2}\right)^{\frac{\varepsilon+2}{2}}}{\left(q^{2}-(1-u)^{2} p^{2}\right)^{\varepsilon+2}}+\frac{(1-u)^{\varepsilon+2}(p)^{\varepsilon+2}}{\left(q^{2}-(1-u)^{2} p^{2}\right)^{\varepsilon+2}}\right]
$$

To evaluate this integral let us consider the standard integral,

$$
\int \frac{\mathrm{d}^{n} q}{(2 \pi)^{n}} \frac{\left(q^{2}\right)^{r}}{\left(q^{2}+c^{2}\right)^{m}}=\frac{1}{\left(16 \pi^{2}\right)^{n / 4}}\left(c^{2}\right)^{\left(\frac{n}{2}\right)+r-m} \frac{\Gamma\left(r+\frac{n}{2}\right) \Gamma\left(m-r-\frac{n}{2}\right)}{\Gamma\left(\frac{n}{2}\right) \Gamma(m)}
$$

Using Equation (3.1.14) in Equation (3.1.13), we get,

$$
\begin{aligned}
I_{1}= & \frac{1}{\left(16 \pi^{2}\right)^{3 / 4}}\left[\left\{-(1-u)^{2} p^{2}\right\}^{\left(\frac{3}{2}+\frac{\varepsilon+2}{2}-(\varepsilon+2)\right)} \frac{\Gamma\left(\frac{\varepsilon+2}{2}+\frac{3}{2}\right) \Gamma\left(\varepsilon+2-\frac{\varepsilon+2}{2}-\frac{3}{2}\right)}{\Gamma\left(\frac{3}{2}\right) \Gamma(\varepsilon+2)}\right. \\
& \left.+(1-u)^{\varepsilon+2}(p)^{\varepsilon+2}\left\{-(1-u)^{2} p^{2}\right\}^{\left(\frac{3}{2}+0-\varepsilon-2\right)} \cdot \frac{\Gamma\left(\frac{3}{2}\right) \Gamma\left(\varepsilon+2-\frac{3}{2}\right)}{\Gamma\left(\frac{3}{2}\right) \Gamma(\varepsilon+2)}\right] \\
= & \frac{1}{(4 \pi)^{3 / 2}} \frac{1}{\Gamma(\varepsilon+2)}\left[(-1)^{\frac{1-\varepsilon}{2}}(1-u)^{1-\varepsilon}(p)^{1-\varepsilon} \frac{2}{\sqrt{\pi}} \Gamma\left(\frac{\varepsilon}{2}+\frac{5}{2}\right) \Gamma\left(\frac{\varepsilon}{2}-\frac{1}{2}\right)\right. \\
& \left.+(-1)^{-\left(\varepsilon+\frac{1}{2}\right)}(1-u)^{1-\varepsilon}(p)^{\varepsilon+2}(p)^{-(2 \varepsilon+1)} \Gamma\left(\varepsilon+\frac{1}{2}\right)\right]
\end{aligned}
$$

Thus Equation (3.1.12) becomes,

$$
\begin{aligned}
\xi_{V V}^{A}(\varepsilon)= & \frac{e^{2} \Gamma(\varepsilon+2)}{2 \Gamma(\varepsilon)} \frac{1}{(4 \pi)^{3 / 2}} \frac{1}{\Gamma(\varepsilon+2)} \int \mathrm{d}^{3} p V(p) V(-p) \int_{0}^{1} \mathrm{~d} u\left[(-1)^{\frac{1}{2}-\frac{\varepsilon}{2}}\right. \\
& \cdot(1-u)^{1-\varepsilon}(p)^{1-\varepsilon} \frac{2}{\sqrt{\pi}} \Gamma\left(\frac{\varepsilon}{2}+\frac{5}{2}\right) \Gamma\left(\frac{\varepsilon}{2}-\frac{1}{2}\right) \\
& \left.+(-1)^{-\left(\varepsilon+\frac{1}{2}\right)}(1-u)^{1-\varepsilon}(p)^{\varepsilon+2}(p)^{-(2 \varepsilon+1)} \Gamma\left(\varepsilon+\frac{1}{2}\right)\right]
\end{aligned}
$$




$$
\begin{aligned}
= & \frac{e^{2}}{8 \pi^{2}} \frac{1}{\Gamma(\varepsilon)} \int \mathrm{d}^{3} p V(p) V(-p)\left[(-1)^{\frac{1}{2}-\frac{\varepsilon}{2}}\left(\frac{1}{2-\varepsilon}\right)\right. \\
& \cdot(p)^{1-\varepsilon}\left(\frac{\varepsilon}{2}+\frac{3}{2}\right)\left(\frac{\varepsilon}{2}+\frac{1}{2}\right)\left(\frac{\varepsilon}{2}-\frac{1}{2}\right)\left\{\Gamma\left(\frac{\varepsilon}{2}-\frac{1}{2}\right)\right\}^{2} \\
& \left.+\frac{\sqrt{\pi}}{2}(-1)^{-\left(\varepsilon+\frac{1}{2}\right)}\left(\frac{1}{2-\varepsilon}\right)(p)^{\varepsilon+2}(p)^{-(2 \varepsilon+1)} \Gamma\left(\varepsilon+\frac{1}{2}\right)\right] \\
= & \frac{e^{2}}{8 \pi^{2}} \int \mathrm{d}^{3} p V(p) V(-p)\left[(-1)^{\frac{1}{2}-\frac{\varepsilon}{2}}\left(\frac{1}{2-\varepsilon}\right)\right. \\
& \cdot(p)^{1-\varepsilon}\left(\frac{\varepsilon}{2}+\frac{3}{2}\right)\left(\frac{\varepsilon}{2}+\frac{1}{2}\right)\left(\frac{\varepsilon}{2}-\frac{1}{2}\right) \frac{\Gamma(\varepsilon)}{4} \frac{1}{\Gamma(\varepsilon)} \\
& \left.+\frac{\sqrt{\pi}}{2}(-1)^{-\left(\frac{\varepsilon}{2}+\frac{1}{2}\right)}\left(\frac{1}{2-\varepsilon}\right)(p)^{\varepsilon+2}(p)^{-(2 \varepsilon+1)} \Gamma\left(\frac{\varepsilon}{2}+\frac{1}{2}\right) \frac{1}{\Gamma(\varepsilon)}\right]
\end{aligned}
$$

where we have used $\left[\Gamma\left(\frac{\varepsilon-1}{2}\right)\right]^{2}=\frac{\Gamma(\varepsilon)}{4}$.

$$
\begin{aligned}
= & \frac{e^{2}}{8 \pi^{2}} \int \mathrm{d}^{3} p V(p) V(-p)\left[(-1)^{\frac{1}{2} \frac{\varepsilon}{2}}(p)^{1-\varepsilon} \frac{\left(\varepsilon^{3}+3 \varepsilon^{2}-\varepsilon-3\right)}{24(2-\varepsilon)}\right. \\
& \left.+\frac{\sqrt{\pi}}{2}(-1)^{-\left(\varepsilon+\frac{1}{2}\right)}\left(\frac{1}{2-\varepsilon}\right)(p)^{\varepsilon+2}(p)^{-(2 \varepsilon+1)} \frac{2^{2 \varepsilon-1}\left(\Gamma\left(\varepsilon+\frac{1}{2}\right)\right)^{2}}{\sqrt{\pi} \Gamma(2 \varepsilon)}\right]
\end{aligned}
$$

[Using Duplication formula]

$$
\begin{aligned}
= & \frac{e^{2}}{8 \pi^{2}} \int \mathrm{d}^{3} p V(p) V(-p)\left[(-1)^{\frac{1}{2}-\frac{\varepsilon}{2}}(p)^{1-\varepsilon} \frac{\left(\varepsilon^{3}+3 \varepsilon^{2}-\varepsilon-3\right)}{24(2-\varepsilon)}\right. \\
& \left.+(-1)^{-\left(\varepsilon+\frac{1}{2}\right)}(p)^{\varepsilon+2}(p)^{-(2 \varepsilon+1)} 2^{2 \varepsilon-1} \frac{(2 \varepsilon+1) \varepsilon}{(2-\varepsilon)}\right]
\end{aligned}
$$

where we have used $\left[\Gamma\left(\frac{2 \varepsilon+1}{2}\right)\right]^{2}=\left(\frac{2 \varepsilon+1}{4}\right) \Gamma(2 \varepsilon+1)$.

To see sign of the term, let us put $\varepsilon \rightarrow 0$ in the factor $(-1)^{\frac{1}{2}-\frac{\varepsilon}{2}}$ and $(-1)^{-\left(\varepsilon+\frac{1}{2}\right)}$, then these terms are equal to $i$ and $-i$. Thus the above equation becomes,

$$
\begin{aligned}
\xi_{V V}^{A}(\varepsilon)= & \frac{i e^{2}}{8 \pi^{2}} \int \mathrm{d}^{3} p V(p) V(-p)\left[(p)^{1-\varepsilon} \frac{\left(\varepsilon^{3}+3 \varepsilon^{2}-\varepsilon-3\right)}{24(2-\varepsilon)}\right. \\
& \left.-(p)^{\varepsilon+2}(p)^{-(2 \varepsilon+1)} 2^{2 \varepsilon-1} \frac{(2 \varepsilon+1) \varepsilon}{(2-\varepsilon)}\right]
\end{aligned}
$$

Now differentiating Equation (3.1.15) w. r. to $\varepsilon$, we get 


$$
\begin{aligned}
\xi_{V V}^{\prime A}(\varepsilon)= & \frac{i e^{2}}{8 \pi^{2}} \int \mathrm{d}^{3} p V(p) V(-p)\left[\frac{1}{24}(p)^{1-\varepsilon} \ln (p)(-1) \frac{\left(\varepsilon^{3}+3 \varepsilon^{2}-\varepsilon-3\right)}{(2-\varepsilon)}\right. \\
& +\frac{1}{24}(p)^{1-\varepsilon}\left\{\frac{(2-\varepsilon)\left(3 \varepsilon^{2}+6 \varepsilon-1\right)-\left(\varepsilon^{3}+3 \varepsilon^{2}-\varepsilon-3\right)(-1)}{(2-\varepsilon)^{2}}\right\} \\
& +(p)^{\varepsilon+2} \ln (p)(p)^{-(2 \varepsilon+1)} 2^{2 \varepsilon-1} \frac{(2 \varepsilon+1) \varepsilon}{(2-\varepsilon)}(p)^{\varepsilon+2}(p)^{-(2 \varepsilon+1)} \ln (p) \\
& +(-2) 2^{2 \varepsilon-1} \frac{(2 \varepsilon+1) \varepsilon}{(2-\varepsilon)}+(p)^{\varepsilon+2}(p)^{-(2 \varepsilon+1)} 2^{2 \varepsilon-1} \ln (2)(2) \frac{(2 \varepsilon+1) \varepsilon}{(2-\varepsilon)} \\
& \left.+(p)^{\varepsilon+2}(p)^{-(2 \varepsilon+1)} 2^{2 \varepsilon-1} \frac{(2-\varepsilon)(4 \varepsilon+1)-\left(2 \varepsilon^{2}+\varepsilon\right)(-1)}{(2-\varepsilon)^{2}}\right]
\end{aligned}
$$

Hence

$$
\begin{aligned}
\xi_{V V}^{\prime A}(0)= & \lim _{\varepsilon \rightarrow 0} \xi_{V V}^{\prime A}(\varepsilon) \\
= & \frac{i e^{2}}{8 \pi^{2}} \int \mathrm{d}^{3} p V(p) V(-p)\left[\frac{1}{24} p \cdot \ln (p)(-1)\left(-\frac{3}{2}\right)\right. \\
& \left.+\frac{1}{24} p \cdot\left(\frac{-5}{4}\right)+(p)^{2} \cdot \frac{1}{p} \cdot \frac{1}{2} \cdot \frac{1}{2}\right] \\
= & \frac{i e^{2}}{128 \pi^{2}} \int \mathrm{d}^{3} p V(p) V(-p) p\left[\ln (p)-\frac{29}{6}\right]
\end{aligned}
$$

Substituting of Equation (3.1.16) into Equation (3.1.11a) yields our final expression for $Z_{1 V V}^{A}$ as,

$$
Z_{1 V V}^{A}=\exp \left[\frac{i e^{2}}{128 \pi^{2}} \int \mathrm{d}^{3} p V(p) V(-p) p\left(\ln (p)-\frac{29}{6}\right)\right]
$$

This contributes to the to the one-loop generating functional for external bosons (photon) lines.

To find one-loop correction for external boson lines from above generating functional, we have to take logarithm on Equation (3.1.17) and then functional differentiation of the expansion with respect to momentum $p$.

Thus the one-loop correction for the external boson lines is,

$$
=\frac{i e^{2}}{128 \pi^{2}} p\left(\ln (p)-\frac{29}{6}\right)
$$

Hence the result in (3.1.18) is finite and of the same form as we obtained by the diagrammatic form of Operator regularization and Dimensional regularization methods in Section-2. In this section we have shown and explained how one can choose the appropriate terms from the Schwinger expansion for the problem in hand.

\subsection{One-Loop Generating Functional and Loop Corrections for External Fermion Lines and Vertex Function}

In this case we focus on the denominator in Equation (3.3), so that let us regu- 
late the $\operatorname{det} B$ through use of the $\xi$-function in Equation (3.11a) yielding

$$
\operatorname{det} B=Z_{1}^{B}=\exp \left[-\frac{1}{2} \lim _{\varepsilon \rightarrow 0} \xi^{\prime B}(\varepsilon)\right] \text {, }
$$

where,

$$
\begin{aligned}
\xi^{B}(\varepsilon)= & \frac{1}{\Gamma(\varepsilon)} \int_{0}^{\infty} \mathrm{d} t t^{\varepsilon-1} \operatorname{tr} \exp \left\{-t\left[p^{2} \delta_{\mu \nu}-\left(1-\frac{1}{\alpha}\right) p_{\mu} p_{v}\right.\right. \\
& \left.\left.-e^{2} \bar{\eta} \gamma_{\mu} \frac{1}{p-e V} \gamma_{\nu} \eta-e^{2} \bar{\eta} \gamma_{\nu} \frac{1}{p-e V} \gamma_{\mu} \eta\right]\right\}
\end{aligned}
$$

In Equation (3.2.2) it is understood that the exponential is $\operatorname{tr}[\exp (-t B)]$, where

$$
\begin{aligned}
B_{\mu \nu} & \equiv p^{2} \delta_{\mu \nu}-\left(1-\frac{1}{\alpha}\right) p_{\mu} p_{v}-e^{2} \bar{\eta} \gamma_{\mu} \frac{1}{p-e V} \gamma_{\nu} \eta-e^{2} \bar{\eta} \gamma_{\nu} \frac{1}{p-e V} \gamma_{\mu} \eta \\
& \equiv B_{0 \mu \nu}+B_{I \mu \nu}
\end{aligned}
$$

where, $B_{0 \mu \nu}$ is independent of the background field $\eta$ and $\bar{\eta}$, and $B_{I \mu \nu}$ is at least linear in $\eta$ and $\bar{\eta}$.

Now as before to use Schwinger expansion in this case let us use the Equation (2.4) and then taking bilinear in $\eta$ and $\bar{\eta}$ on the on the right-hand side of Equation (3.2.1), we end up with

$$
\begin{aligned}
Z_{1}^{B}= & \exp \left[-\frac{1}{2}\left\{\operatorname { l i m } _ { \varepsilon \rightarrow 0 } \frac { \mathrm { d } } { \mathrm { d } \varepsilon } \left(\frac { 1 } { \Gamma ( \varepsilon ) } \int _ { 0 } ^ { \infty } \mathrm { d } t t ^ { \varepsilon } \operatorname { t r } \left\{\exp \left(p^{2} \delta_{\mu \nu}-\left(1-\frac{1}{\alpha}\right) p_{\mu} p_{\nu}\right)\right.\right.\right.\right. \\
& \left.\left.\left.\cdot t\left(-2 e^{2} \bar{\eta} \gamma_{\mu} D^{-1} \gamma_{\nu} \eta\right)\right\}\right\}\right]
\end{aligned}
$$

The exponential factor in the trace of Equation (3.2.4) can be simplified using the complete set of orthonormal projections operators:

$$
\begin{gathered}
T_{\mu v}(p)=\left(\delta_{\mu v}-\frac{p_{\mu} p_{v}}{p^{2}}\right) \\
L_{\mu \nu}(p)=\frac{p_{\mu} p_{v}}{p^{2}}
\end{gathered}
$$

These allows us to write $\left(e^{-t B_{0}}\right)_{\mu \nu}$ as

$$
\begin{aligned}
& \exp \left(p^{2} \delta_{\mu v}-\left(1-\frac{1}{\alpha}\right) p_{\mu} p_{v}\right) t \\
& =\sum_{n=0}^{\infty} \frac{1}{n !}\left[p^{2} t\left(T_{\mu v}+\frac{1}{\alpha} L_{\mu v}\right)\right]^{n}=e^{-t p^{2}} T_{\mu v}+e^{-t p^{2} / \alpha} L_{\mu v}
\end{aligned}
$$

and let us expand $\mathbb{D}^{-1}$ in powers of the back-ground field in the $\xi$-function Equation (3.2.4):

$$
\begin{aligned}
D^{-1} & =\frac{1}{p-e V}=\frac{1}{p}\left(1-e V \frac{1}{p}\right)^{-1} \\
& =\left(\frac{1}{p}+\frac{1}{p} e V \frac{1}{p}+\frac{1}{p} e V \frac{1}{p} e V \frac{1}{p}+\cdots\right)
\end{aligned}
$$


It is interesting to note that at this stage this is straightforward to apply the perturbative expansion of Equation (2.3) to this $\xi$-function and to select from the expansion those terms appropriate for any particular Greens function. This means that from the expansion we can choose appropriate terms that are associated with the related problems that we are interested in. Let us consider here the $\xi$-function for the fermion two-point function and the vertex function, we find

$$
\xi^{B}(\varepsilon) \cong \frac{e^{2}}{\Gamma(\varepsilon)} \int_{0}^{\infty} \mathrm{d} t t^{\varepsilon} \operatorname{tr}\left[\bar{\eta} \gamma_{\mu}\left(\frac{1}{p}+\frac{1}{p} e V \frac{1}{p}\right) \gamma_{\nu} \eta\left(e^{-t p^{2}} T_{\mu \nu}+e^{-t p^{2} / \alpha} L_{\mu \nu}\right)\right]
$$

Following ref. [15] [16] in the Approach-A, we compute from Equation (3.2.7) the $\xi$-function in the limit of zero momentum transfer to the photon:

$$
\begin{array}{r}
\xi^{B}(\varepsilon)=\frac{e^{2}}{8 \pi^{2}} \int \mathrm{d}^{3} p\left(\frac{1}{p^{2}}\right) \frac{\Gamma(1-\varepsilon)}{\Gamma(3-\varepsilon)}\left[2+\left(\alpha^{\varepsilon+1}-1\right) \frac{(2-\varepsilon)}{(1+\varepsilon)}\right] \\
\cdot \bar{\eta}(p)\left[p+\frac{e}{4 \pi^{2}}\left(V(0)-2 \varepsilon \frac{p}{p^{2}} p \cdot V(0)\right)\right] \eta(-p)
\end{array}
$$

Therefore by Equation (3.2.1) the contributions to the one-loop generating functional is

$$
\begin{aligned}
Z_{1}^{B} \cong & \exp \left\{\frac { 1 } { 2 } \left[\frac{e^{2}}{8 \pi^{2}} \int \mathrm{d}^{3} p\left(\frac{3}{2}+\alpha \ln \alpha-\alpha \ln p^{2}\right) \bar{\eta}(p)\left(p+\frac{e}{4 \pi^{2}} V(0)\right) \eta(-p)\right.\right. \\
& \left.\left.-\alpha \frac{e^{3}}{16 \pi^{4}} \int \mathrm{d}^{3} p \bar{\eta}(p) p \eta(-p) \frac{p \cdot V(0)}{p^{2}}\right]\right\}
\end{aligned}
$$

This contributes to the one-loop generating functional for external fermion (electron) lines and vertex function in QED.

To obtain the one-loop correction for external fermion lines and vertex function, we have to take logarithm of Equation (3.2.9) and then functional differentiation with respect to momentum $p$.

Hence from Equation (3.2.9), we get

$$
\begin{aligned}
= & \frac{e^{2}}{16 \pi^{2}}\left(\frac{3}{2}+\alpha \ln \alpha-\alpha \ln p^{2}\right) \bar{\eta}(p) p \eta(-p) \\
& +\frac{e^{3}}{64 \pi^{4}}\left(\frac{3}{2}+\alpha \ln \alpha-\alpha \ln p^{2}\right) \bar{\eta}(p) V(0) \eta(-p) \\
& -\alpha \frac{e^{3}}{32 \pi^{4}} \bar{\eta}(p) \gamma^{\mu} p_{\mu} \eta(-p) \frac{p^{v} V_{v}(0)}{p^{2}} \\
= & \frac{e^{2}}{16 \pi^{2}}\left(\frac{3}{2}+\alpha \ln \alpha-\alpha \ln p^{2}\right) \bar{\eta}(p) p \eta(-p) \\
& +\frac{e^{3}}{64 \pi^{4}}\left(\frac{3}{2}+\alpha \ln \alpha-\alpha \ln p^{2}-2 \alpha\right) \bar{\eta}(p) V(0) \eta(-p)
\end{aligned}
$$

From the expansion (3.2.10) we can find the one-loop correction for the external fermion lines and one-loop vertex function by choosing the appropriate terms. This expression is of the same form as obtained by DR and OR methods 
with Feynman diagrams in Section-2.

Thus the one-loop correction for the external fermion lines is,

$$
\frac{e^{2}}{16 \pi^{2}} p\left(\frac{3}{2}+\alpha \ln \alpha-\alpha \ln p^{2}\right)
$$

and the one-loop correction to the vertex function is,

$$
\frac{e^{3}}{64 \pi^{4}}\left(\frac{3}{2}+\alpha \ln \alpha-\alpha \ln p^{2}-2 \alpha\right)
$$

The result in (3.2.11) and (3.2.12) is of the same form as we obtained by the diagrammatic form of Operator regularization and Dimensional regularization methods in Section-2.

\section{Conclusion}

Radiative corrections in quantum field theory are very important for renormalization of a theory. Because when we evaluate loop-diagrams in some cases we find both finite and infinite terms. To absorb the infinite parts is the renormalization. That is why best regularization method is needed to get the correct finite and infinite parts of the loop-diagrams. Dimensional regularization (DR) is one of the best methods to evaluate the loop diagrams, but it has also some limitations in some cases. Operator regularization (OR) method had been prescribed to overcome some of these problems. To compare the advantage of OR, we have evaluated basic loop diagrams in QED using OR and compared the result with DR. We found that the result is finite and comparable, which was expected. In an earlier paper also we have shown the comparable result between OR and DR where the result consists of finite and divergent parts. From these calculations it is clear that $\mathrm{OR}$ is an attractive method to use in evaluating loop diagrams.

\section{Conflicts of Interest}

The authors declare no conflicts of interest regarding the publication of this paper.

\section{References}

[1] Yang, C.N. and Mills, R.L. (1954) Convervation of Isotopic Spin and Isotopic Gauge Invariance. Physical Review Journals Archive, 96, 191. https://doi.org/10.1103/PhysRev.96.191

[2] Glashow, S.L. (1961) Partial Symmetries of Weak Interaction. Nuclear Physics, 22, 579-588. https://doi.org/10.1016/0029-5582(61)90469-2

[3] Weinberg, S. (1967) A Model of Leptons. Nuclear Physics, 19, 1264.

[4] Salam, A. (1968) Elementary Particle Theory. In: Svartholm, N., Ed., Weak and Electromagnetic Interactions, Almquist Wiksell, Stockholm.

[5] Glashow, S.L., Illiopulos, J. and Maiani, I. (1970) Weak Interactions with Lepton-Hadron Symmetry. Physical Review D, 2, 1285. https://doi.org/10.1103/PhysRevD.2.1285

[6] Salam, A. and Strathdee, J. (1975) Transition Electromagnetic Fields in Particle Physics. Nuclear Physics B, 90, 203. https://doi.org/10.1016/0550-3213(75)90642-2 
[7] Dowker, J. and Critchley, R. (1976) Effective Langrangian and Energy-Momentum Tensor in De Sitter Space. Physical Review D 13, 3224. https://doi.org/10.1103/PhysRevD.13.3224

[8] Hooft, G.'t and Veltman, M. (1972) Regularization and Renormalization of Gauge Fields. Nuclear Physics B, 44, 189. https://doi.org/10.1016/0550-3213(72)90279-9

[9] Garcial-Aguilar, J.D. and Perez-Lorenzana, A. (2015) Dimensional Regularization with Non Beta-Functions. Revista Mexicana de Fisica, 61, 149.

[10] Pauli, W. and Villars, F. (1949) On the Invariant Regularization in Relativistic Quantum Theory. Reviews of Modern Physics, 21, 434. https://doi.org/10.1103/RevModPhys.21.434

[11] Chowdhury, A.M., et al. (1985) Preregularization. Canadian Journal of Physics, 63, 1453. https://doi.org/10.1139/p85-244

[12] Chowdhury, A.M. and McKeon, D.G.C. (1986) Surface Terms and Radiative Corrections to the VVA Triangle Diagrams. Physical Review D, 33, 598. https://doi.org/10.1103/PhysRevD.33.598

[13] Chowdhury, A.M., McKeon, D.G.C. and Mann, R.B. (1986) Ambiguities of Chairal-Anomaly Graph in Higher Dimensions. Physical Review D, 33, 3090. https://doi.org/10.1103/PhysRevD.33.3090

[14] Chowdhury, A.M., et al. (1986) Preregularization and the Structure of Loop Momentum Ambiguities within Quantum Corrections to the Supercurrent. Physical Review D, 34, 619. https://doi.org/10.1103/PhysRevD.34.619

[15] McKeon, D.G.C. and Sherry, T.N. (1987) Operator Regularization of Green's Functions. Physical Review Letters, 59, 532. https://doi.org/10.1103/PhysRevLett.59.532

[16] McKeon, D.G.C. and Sherry, T.N. (1987) Operator Regularization and One-Loop Green's Functions. Physical Review D, 35, 3854-3872. https://doi.org/10.1103/PhysRevD.35.3854

[17] Chowdhury, A.M. and McKeon, D.G.C. (1987) Operator Regularization in Weinberg-Salam Model. Canadian Journal of Physics, 65, 1082-1089. https://doi.org/10.1139/p87-179

[18] Shiekh, A.Y. (2010) Operator Regularization of Feynman Diagrams in One Loop QED.

[19] Shiekh, A.Y. (2011) Operator Regularization of Feynman Diagrams at Multi-Loop Order.

[20] Forkan, M. and Abul Mansur Chowdhury, M. (2012) Operator Regularization in Evaluating Feynman Diagrams in QED in $(3+1)$ Dimensional Space-Time. IOSR Journals, 3, 36-51.

[21] De Witt, B. (1967) Quantum Theory of Gravity. II. The Manifest Covariant Theory. Physical Review, 162, 1195-1239. https://doi.org/10.1103/PhysRev.162.1195

[22] Kallosh, R.E. (1974) The Renormalization in Non-Abelian Gauge Fields. Nuclear Physics B, 78, 293-312. https://doi.org/10.1016/0550-3213(74)90284-3

[23] Abbott, L.F. (1981) The Background Fields Method beyond One-Loop. Nuclear Physics B, 185, 189-203. https://doi.org/10.1016/0550-3213(81)90371-0

[24] Hawking, S. (1977) Zeta Function Regularization of Path Integral in Curved Space. Communications in Mathematical Physics, 55, 133-148. https://doi.org/10.1007/BF01626516

[25] Reuter, M. (1985) Chiral Anomalies and Zeta-Function Regularization. Physical Review D, 31, 1374-1385. https://doi.org/10.1103/PhysRevD.31.1374 
[26] Schwinger, J. (1951) On Gauge Invariance and Vacuum Polarization. Physical Review, 82, 664-679. https://doi.org/10.1103/PhysRev.82.664

[27] Ryder, L.H. (1987) Quantum Field Theory. Cambridge University Press, Cambridge.

[28] Ramond, P. (1981) Field Theory: A Modern Primer. Benjamin/Cummings.

[29] Lee, T.D. (1981) Particle Physics and Introduction Field Theory. Harwood Academic Publisher, Amsterdam. 